\title{
Caminando hacia la educación inclusiva en Aragón
}

\section{Walking towards inclusive education in Aragon} Resumen: El Centro Aragonés de Recursos para la Edu-
cación Intercultural es una iniciativa del Departamento de
Educación, Universidad, Cultura y Deporte, al que corres-
ponde su titularidad. Se crea por el Decreto $281 / 2002$ de 3
de septiembre, y se regula mediante la Orden de 11 de julio
de 2007 , del Departamento de Educación, Cultura y Depor-
te, donde se aprueba su organización y funcionamiento. Su
dependencia funcional se atribuye a la Dirección General de
Política Educativa y Educación Permanente, que cuenta en-
tre sus competencias la gestión de la educación permanente
y formación del profesorado, así como la innovación y orien-
tación educativa de la Comunidad Autónoma de Aragón. En
la actualidad, el Centro Aragonés de Recursos está dando
un giro hacia la educación inclusiva, siendo el referente de
este tema en Aragón. Próximamente se tiene previsto que
se regule en una nueva Orden su estructura, organización y
funcionamiento, así como su ámbito de actuación.
Palabras-clave: Educación inclusiva. Aragón. Políticas Educativas.

\begin{abstract}
The Aragon Resources Center for the Intercultural Education is an initiative of the Education, University, Culture and Sports Department, which also has its ownership. The Center is created by the 281/2002 decree of 3 September and it is regulated through the Education, Culture and Sports Department's order of 11 July 2007, that approves its organization and functioning. Its functional dependency is attributable to the General Educational Politics and Permanent Education Direction, that counts among its competencies with the management of education and the formation of faculty, as well as the educational innovation and orientation in the Autonomous Community of Aragon. Nowadays, the Aragon Resources Center is shifting towards inclusive education, being the reference for this matter in Aragon. A new Order regulating its structure, organization and functioning as well as its procedures and sphere of activity, is expected soon.

Keywords: Inclusive Education. Aragon. Educational Politics.
\end{abstract}

CARMONA, Coral Elizondo. Caminando hacia la educación inclusiva en Aragón. Informática na Educação: teoria e prática, Porto Alegre, v. 16, n. 2, p. 17.-26, jul./dez. 2013.

\section{Coral Elizondo Carmona}

CAREI

\section{Un poco de historia}

5 los años 90, España pasa de ser un país de emigración a ser un país de inmigración, un país de acogida; esto se debe fundamentalmente al desarrollo económico y social que se experimenta en aquel momento en España. Esta primera inmigración proviene fundamentalmente de países comunitarios como Rumanía, Polonia y Bulgaria y no comunitarios, como el Magreb, Argelia y África subsahariana.

En aquel momento, los primeros trabajadores extranjeros eran varones que venían a trabajar al campo aragonés, y que no estaban acompañados por su familia, por lo que en las aulas de los centros educativos, apenas teníamos alumnado inmigrante.

A principios del siglo XXI, la llegada de población extranjera se multiplicó por 6,5 y este crecimiento poblacional supuso que los centros educativos aragoneses comenzaran a recibir en sus aulas por primera vez alumnado con desconocimiento del idioma, alumnado de culturas y creencias diferentes. Cuestiones como 
lengua, nacionalidad, cultura o religión, serán a partir de entonces variables a tener en cuenta en los centros educativos cuando se hable de diversidad.

España aún no tenía contemplado en sus leyes este hecho, que comenzaba a ser patente en los centros educativos; habría que esperar hasta el año 2006 con la Ley Orgánica 2/2006, de 3 de mayo, de Educación, para que este alumnado que se incorporaba tardíamente al sistema educativo por proceder de otros países y que requería una atención diferente a la ordinaria, recibiera la atención educativa necesaria, de forma que las enseñanzas que ofrecía el sistema educativo se pudieran adaptar para dar respuestas a las necesidades específicas de apoyo de este alumnado. Dicha adaptación garantizaría su acceso, permanencia y progresión en el sistema.

En este contexto, el Gobierno de Aragón, que tenía transferencias en educación, creó por el Decreto 281/2002 de 3 de septiembre, el Centro Aragonés de Recursos para la Educación Intercultural, CAREI, un centro al servicio de la comunidad educativa aragonesa para todos los temas relacionados con la acogida e integración de la población de origen inmigrante en el contexto educativo desde la óptica de la interculturalidad. La creación de este centro constituyó una apuesta de futuro abierta a construir caminos de encuentro y mestizaje, y que llevaban a que Aragón fuera una sociedad más libre, justa y solidaria.

Esta apuesta del Gobierno de Aragón del año 2002 ya fue en su momento una postura importante hacia la inclusión, entendida como el desarrollo de escuelas que acogen a todo el alumnado, independientemente de su pertenencia a minorías étnicas o grupos culturales minoritarios, o cualesquiera que sean sus características o dificultades individuales.

\section{Concepto de educación inclusiva. Qué entendemos por inclusión}

El origen de la idea de inclusión se sitúa en el Foro Internacional de la UNESCO, que durante años viene formulando y aplicando políticas orientadas hacia una Educación Inclusiva, y desde donde la inclusión surge como un movimiento orientado a transformar los sistemas educativos para responder a la diversidad del alumnado, como un avance frente al concepto de integración. "Esto supone que la educación inclusiva tenga por objeto eliminar la exclusión social como consecuencia de actitudes y respuestas a la diversidad en términos de raza, clase social, origen étnico, religión, género y aptitudes... asegurando que la Educación Para Todos signifique realmente todos" ${ }^{11}$, tal y como quedó patenten en la Conferencia Internacional de Educación (CIE) en su 48 a reunión celebrada en Ginebra en noviembre de 2008.

El concepto de una educación de calidad y equidad deben ir unidos al de educación inclusiva. En la Ley Orgánica de Educación se habla en su Preámbulo de "mejorar el nivel educativo de todo el alumnado, conciliando la calidad de la educación con la equidad de su reparto". También se habla "del principio de esfuerzo compartido aplicable a todos los miembros de la comunidad educativa, realizando cada uno de ellos una contribución específica".

La UNESCO define la educación inclusiva como "un proceso orientado a responder la diversidad de los estudiantes incrementando su participación y reduciendo la exclusión en y desde la educación. Está relacionada con la presencia, la participación y los logros de todos los alumnos, con especial énfasis en aquellos

1 (UNESCO, Educación inclusiva: el camino hacia el futuro, 2009). 
que, por diferentes razones están excluidos o en riesgo de ser marginados".2

Nos quedamos con estas palabras clave para entender bien el concepto; proceso, puesto que la educación inclusiva no es algo estático, no termina cuando hemos concluido una práctica; debemos desarrollar en los centros educativos culturas y prácticas inclusivas dinámicas, que se puedan modificar, que estén adaptadas a nuestro contexto, a la realidad de nuestro centro, a nuestras aulas; el avance hacia la inclusión, sólo se puede producir por la reducción significativa de la exclusión; para Ainscow (2005) "la inclusión debe ser considerada como una búsqueda interminable de formas más adecuadas de responder a la diversidad"; presencia, "se refiere al acceso y permanencia en la escuela $u$ otras modalidades formales" (BLANCO, 2008, pág. 7), pero la educación inclusiva va más allá del acceso, una educación inclusiva de calidad tiene en cuenta las capacidades y potencialidades de cada persona con el fin de desarrollarlas al máximo; participación, "significa que el currículo y las actividades educativas contemplen las necesidades de todos los estudiantes" (BLANCO, 2008, pág. 7); quiere decir que todo el alumnado del aula pueda llevar a cabo la actividad que estemos desarrollando, que pueda participar en ella, que pueda aprender, que tenga algo que aportar; en este sentido hablamos de una educación de calidad para todos, pero también "incluye, inevitablemente, la opinión de los propios alumnos" (AINSCOW, 2005, p. 30); debemos, por lo tanto, dar voz y visibilidad a todo el alumnado; los logros "hacen referencia a la necesidad de que todos los estudiantes adquieran, en la medida de sus posibilidades, los aprendizajes establecidos en el

2 (UNESCO, Orientaciones para la Inclusión: asegurar el acceso a la educación para todos, 2006). currículo escolar, necesarios para su desarrollo personal y socialización" (BLANCO, 2008, p. 7).

Muntaner (2012) cuando habla de Educación Inclusiva, habla de "tres pes", que se van a corresponder con los conceptos anteriores: participación, presencia y progreso, entendida esta última como logro.

\section{Caminando hacia una educación inclusiva en Aragón. CAREI}

En estos momentos, y durante todo el curso escolar 2012/2013, el Centro Aragonés de Recursos, CAREI, ha dado un giro hacia la educación inclusiva, y pasará a denominarse Centro Aragonés de Recursos para la Educación Inclusiva, convirtiéndose en un centro de referencia para la comunidad educativa de Aragón y del estado Español en materia de educación inclusiva. Otra vez más, la Comunidad Autónoma de Aragón apuesta con fuerza hacia una educación de calidad para todos con la nueva orientación dada al centro, que próximamente se tiene prevista regular en una nueva Orden.

EI CAREI es un centro de recursos, tanto humanos como materiales, al servicio de todos los centros educativos de Aragón. Desde el CAREI se crea, diseña, gestiona y mantiene actualizado un centro de recursos didácticos relacionado con la educación inclusiva para los diversos niveles y etapas del sistema educativo en Aragón; se ofrecen respuestas educativas a todas las demandas que puedan surgir en los centros educativos relacionadas con el español como lengua extranjera, con mediación intercultural o con la educación inclusiva en general; se mantienen relaciones de colaboración e intercambio de la información con aquellas instituciones u organizaciones del es- 
tado o del exterior que persigan fines similares en sus respectivos ámbitos educativos; se desarrollan actividades encaminadas a potenciar el desarrollo de las capacidades a las familias para el cuidado y la educación de sus hijos, contribuyendo de esta forma al desarrollo personal y social de los niños y niñas desde el hogar; pero también se lleva a cabo la formación permanente especializada del profesorado de programas institucionales con ámbito autonómico, programas encaminados a conseguir una educación más inclusiva donde los valores compartidos para atender y dar respuesta en Aragón a la diversidad del alumnado permitan mejorar el aprendizaje, la participación y la presencia de todo el alumnado de los centros.

\subsection{Programas propios del CAREI}

En la actualidad el CAREI cuenta con los siguientes programas.

\section{a) Programa de mediación intercultural}

EI CAREI coordina un equipo humano de mediadores interculturales repartidos por toda la Comunidad Autónoma de Aragón que trabajan para que la convivencia intercultural sea una realidad en todos los centros educativos. El objetivo de este programa de mediación intercultural es lograr un acercamiento entre personas de distintas culturas por medio de una tercera que es el mediador, con la finalidad de crear nuevos marcos de convivencia a través de la comunicación y las relaciones entre personas y grupos. Su objetivo no se queda sólo en la mediación puntual sino que pretende contribuir a enseñar formas de hacer y capacitar al profesorado en el conocimiento de otras culturas y en la creación de dinámicas que favorezcan la autonomía del centro para abordar estos temas.

El equipo humano está formado por profesionales nativos del Magreb, del África subsahariana, de Europa del Este y de China; estos mediadores son mucho más que meros traductores o intérpretes, son puentes que acercan culturas diferentes para conseguir que la convivencia, en el mismo tiempo y espacio, sea una realidad.

Con este programa se persiguen tres objetivos específicos claros:

- Favorecer, desde el punto de vista intercultural, la atención a los alumnos que desconocen el entorno educativo y ayudarles en la integración en el centro.

- Prevenir posibles conflictos debidos a factores culturales e impulsar cambios que promuevan y mejoren la convivencia escolar.

- Actuar para iniciar, mejorar o restablecer la comunicación del centro y las familias afectadas por la existencia de dificultades debidas a diferencias culturales o derivadas del proceso migratorio.

\section{b) Programa de refuerzo de español en horario extraescolar}

El Programa de Refuerzo de Español es un servicio coordinado por el CAREI y dirigido al alumnado con desconocimiento del idioma e incorporación tardía al sistema educativo español de todos los centros educativos públicos y concertados de Educación Secundaria de la ciudad de Zaragoza; hablamos, por lo tanto, del alumnado entre 13 y 17 años. Es importante señalar aquí que todos los institutos de 
educación secundaria y colegios de educación primara de Aragón, que lo soliciten y tengan la necesidad, llevan a cabo dentro de su horario lectivo actividades de enseñanza y aprendizaje del español como segunda lengua para todo el alumnado que lo precise, organizado y llevado a cabo por el propio profesorado del centro. Este programa de refuerzo que coordina el CAREI, es como su propia palabra indica, un refuerzo al trabajo de los centros, un refuerzo que persigue una adquisición de la competencia lingüística.

Este programa se desarrolla en horario de tarde y fuera del horario escolar entre los meses de octubre a mayo. Se enseña el español como segunda lengua siempre desde un enfoque comunicativo y curricular y se potencia que la inclusión de todo el alumnado sea una realidad. Si queremos que este alumnado más vulnerable pueda participar en las actividades del centro, permanecer en el mismo y obtener éxito escolar, es necesario que en primer lugar conozca el idioma.

\section{c) Escuela de familias}

La familia y la escuela son sistemas abiertos que tienen funciones diferentes, pero complementarias. "El proceso de socialización se inicia en la familia y continúa y se complementa en la escuela" (MIR; BATLE; HERNÁNDEZ, 2009). Es necesario, por lo tanto, que familia y escuela caminemos en la misma dirección y que se fomente la implicación, la participación y la responsabilidad de las familias en el proyecto educativo del centro.

El objetivo fundamental que se persigue con este programa es potenciar el desarrollo de las capacidades de los padres para el cuidado y la educación de sus hijos; y de esta forma contribuir al desarrollo personal y social de los niños y niñas desde la propia familia.

Para conseguir dicho objetivo se pretende ofrecer a las familias la información y conocimientos básicos sobre diferentes temas; así como facilitar recursos educativos y formativos para que las familias puedan promover en sus hijos e hijas actitudes, valores, habilidades personales y sociales sanas, que les permitan afrontar, de manera responsable, la vida. Sin olvidarnos de encontrar espacios donde promover el intercambio de experiencias entre todas las familias asistentes, incidiendo en el intercambio y enriquecimiento de las diferentes culturas que se agrupan en las comunidades educativas de nuestros centros.

El programa de Escuelas de Familias que dinamiza el CAREI, está abierto a todos los centros educativos de la Comunidad Autónoma y se oferta en módulos cerrados, cuyos contenidos estarán dirigidos a padres y madres del alumnado de Educación Infantil (3 a 6 años), Educación Primaria (6 a 12 años) y Educación Secundaria (12 a 16 años). Entre los contenidos a trabajar se ofertan temas como la comunicación en el hogar, habilidades de comunicación; el desarrollo emocional, autoconciencia, tipos de emociones, vínculo o apego, relaciones sociales positivas; estilos educativos y sus consecuencias, normas y límites; premios y castigos...

Esta actividad se desarrolla a lo largo de un curso escolar en horario de tarde en el propio centro educativo y con una periodicidad mensual y es impartida por psicólogos o psicopedagogos que se desplazan al centro educativo.

\section{d) ARASAAC}

El Portal Aragonés de la Comunicación Aumentativa y Alternativa es coordinado por el CAREI desde el curso 2012/2013, puesto que 
todas sus actuaciones y líneas de trabajo e investigaciones están relacionadas con la educación inclusiva.

Los objetivos de este programa son ampliar, difundir y actualizar el banco de pictogramas que sirven de soporte e instrumento facilitador en los procesos de comunicación al alumnado con barreras para la comunicación que presenta diferentes tipos de discapacidad y para la sociedad en general; creación, de herramientas on-line que faciliten tanto a los profesionales como a las familias, la elaboración de sus propios recursos; creación de sencillos juegos que permitan incluir el juego como objetivo de trabajo tanto en la escuela como en el hogar; difundir los materiales elaborados por los usuarios y mantener relaciones de colaboración con distintas instituciones u organismos del estado con el fin de desarrollar aplicaciones o proyectos comunes.

\subsection{Formación permanente espe- cializada para el profesorado}

En la comunicación que la Comisión Europea hizo al Parlamento Europeo, al Consejo, al Comité Económico y Social Europeo y al Comité de las Regiones sobre un nuevo concepto de educación, en concreto sobre la necesidad de invertir en las competencias para lograr mejores resultados económicos, se habla de "la exigencia de nuevas competencias tanto al profesorado, como a los formadores del profesorado y al personal directivo" (EUROPEA, 2012, pág. 12) de los centros educativos, y vista esta importancia, el CAREI trabaja en tres ámbitos o áreas de trabajo, relacionadas a su vez con las competencias profesionales docentes.

A. Ámbito de Habilidades para la vida. Relacionada con la competencia social-relacional
(Equidad, habilidades sociales, habilidades relacionales, gestión de la participación) y la competencia intra e interpersonal (Habilidades sociales, acción tutorial, orientación, gestión y promoción de valores). En este ámbito se incluyen actividades formativas y de intervención en centros educativos desarrolladas en colaboración con el Departamento de Sanidad, Bienestar Social y Familia del Gobierno de Aragón.

B. Ámbito de convivencia y participación. Relacionada con la competencia en gestión de la convivencia (promoción de la convivencia, mediación y resolución de conflictos, control de la convivencia). Se trabaja en colaboración con la Asesoría de Convivencia del Departamento de Educación, Universidad, cultura y Deporte del Gobierno de Aragón, que atiende y orienta individualmente a todos los miembros de la comunidad educativa (alumnado, familias y profesionales) en casos relacionados con la convivencia y conflictos escolares.

C. Ámbito de orientación y aprendizaje. Relacionada con la competencia ejecutiva: gestión del conocimiento, procedimientos y métodos de investigación, conocimiento curricular, establecimiento de metas.

Dentro de cada uno de estos ámbitos o áreas de trabajo, se llevan a cabo distintas actuaciones que lo desarrollan, que en ocasiones serán cursos de formación especializada y permanente para el profesorado (que se desprenden de programas o actividades institucionales) y en otras intervenciones por medio de proyectos o programas propios del CAREI.

La formación permanente del profesorado en Aragón, así como su régimen jurídico y la estructura de su red, está regulada por el Decreto 105/2013, de 11 de junio, del Gobierno de Aragón. En él se establece que "la formación permanente del profesorado dirigirá sus accio- 
nes a promover un desarrollo profesional que faculte al profesorado para un mejor ejercicio de su práctica docente, con especial atención a: capacitar el profesorado para el desarrollo de los planes institucionales que promueva el Departamento competente en materia de educación (artículo 2.c); desarrollar modelos que permitan al docente introducir mejoras en su práctica educativa y establecer fórmulas de colaboración con sus compañeros (art. 2.d); buscar nuevas y más eficaces formas de desarrollar los contenidos curriculares que imparte su práctica docente a través de la innovación y de la investigación (art. 2.e) y fomentar la formación para la convivencia escolar y para la colaboración entre la escuela, las familias, el alumnado y el entorno social (art. 2.g)"

El CAREI "colabora en la planificación de la formación permanente" y "elabora, desarrolla y evalúa cada curso escolar su Plan de Actuación, que recoge el conjunto de medidas previstas para desarrollar el Plan Anual de Formación Permanente del Profesorado" 3 que persigue:

a) Capacitar al profesorado para el desarrollo de los planes institucionales que promueve la dirección general de política educativa y educación permanente

Desde el CAREI se gestiona la formación del Programa Desarrollo de Capacidades por el que se amplía y enriquece la atención educativa del alumnado que destaca por su elevado rendimiento escolar o por su capacidad o talento especial en algún área y competencia del currículo. Este programa se desarrolla a través de un proyecto de centro y forma parte

\footnotetext{
${ }^{3}$ Artículo 12.c, del Decreto 105/2013, de 11 de junio, del Gobierno de Aragón, por el que se regula el sistema aragonés de formación permanente del profesorado, su régimen jurídico y la estructura de su red.
}

del Plan de Atención a la Diversidad de éste, debe además contribuir a la mejora de la respuesta educativa del centro a la totalidad de su alumnado, incorporando innovaciones metodológicas que hagan que el centro camine hacia una educación inclusiva.

Los Centros de escolarización preferente de alumnos con trastorno del espectro autista, se conciben como un recurso educativo normalizado especializado en la promoción del desarrollo, aprendizaje y participación de este alumnado, proporcionando atención educativa en las etapas correspondientes al segundo ciclo de educación infantil, en la educación primaria y en la educación secundaria obligatoria. Estos centros cuentan con el asesoramiento y apoyo de los servicios de orientación, así como de la formación especializada para todo el profesorado organizada por el CAREI cada curso escolar.

Se proporciona también el asesoramiento en el uso de emisoras de $F M$, para el profesorado que tiene en sus aulas un niño o una niña que utiliza estas emisoras, que son propiedad del Gobierno de Aragón y que se ceden en préstamo al alumnado que las necesita, así como formación para el profesorado que tiene en sus aulas alumnado con discapacidad visual.

\section{b) Introducir mejoras en su práctica docente por medio de formación especia- lizada por su temática}

"La formación permanente del profesorado debe propiciar y facilitar el desarrollo de los procesos continuos de reconstrucción del saber profesional, necesarios para responder a las demandas de la sociedad y al desafío personal y social que cada docente debe asumir para realizarse en plenitud, y vivir ética y 
profesionalmente comprometido con la tarea educativa"4 , para ello, el CAREI organiza los siguientes cursos de formación abiertos a todos los docentes de Aragón: Curso de Claves culturales, donde los mediadores interculturales del centro proporcionan claves, recursos y conocimientos de los distintos colectivos que viven en la Comunidad Autónoma con el fin de trabajar la interculturalidad en todos los centros educativos; Curso de enseñanza del español como segunda lengua, impartido por las profesoras del Programa de Refuerzo de Español y destinado a todo el profesorado, el objetivo es dar a conocer recursos y estrategias para la enseñanza del español al alumnado no alfabetizado o poco escolarizado; ARASAAC. Portal Aragonés de la comunicación Aumentativa y Alternativa. Software, herramientas y materiales para la comunicación, este curso va dirigido a todo el profesorado de Educación Infantil, de Educación Especial y primeros ciclos de Educación Primaria; Jornadas de primeros auxilios y atención al alumnado con enfermedades crónicas, es impartido por sanitarios del Gobierno de Aragón y como destinatarios están todos los docentes y no docentes que trabajen en centros educativos de Aragón y por último la formación relacionada con la convivencia y el absentismo escolar. Dentro del apartado de Salud mental y educación, el CAREI organiza cursos para el profesorado del Centro de Salud Mental Infanto-Juvenil, así como todos aquellos relacionados con esa temáticas y dirigidos a todo el profesorado interesado.

\footnotetext{
${ }^{4}$ Decreto 105/2013, de 11 de junio, del Gobierno de Aragón, por el que se regula el sistema aragonés de formación permanente del profesorado, su régimen jurídico y la estructura de su red.
}

\section{c) Reflexionar conjuntamente por me- dio de seminarios de trabajo}

Los seminarios permiten al profesorado que asiste, profundizar en el estudio de determinados aspectos educativos, reflexionar de forma conjunta sobre temas de interés común, debatir internamente o intercambiar experiencias, además de contar con la colaboración ocasional de personas expertas que ayuden e profundizar sobre determinados aspectos concretos.

Desde el CAREI se coordinan los siguientes seminarios abiertos a todo el profesorado: Seminario autonómico de centros específicos de Educación Especial y Seminario de orientadores educativos. Este último formado por profesores de educación secundaria de la especialidad de orientación educativa, que o bien trabajan en los Equipos de Orientación Educativa y Psicopedagógica (EOEP) que atienden los Centros de Educación Infantil y Primaria o bien en el departamento de orientación de los Institutos de Educación Secundaria.

En el CAREI se coordina y planifica el Seminario autonómico de Educación Inclusiva formado por:

- Los cuatro asesores del CAREI, entre los que se incluye la directora.

- Los seis asesores de la red de formación de las tres provincias de Aragón y que desarrollan esta línea en su ámbito de trabajo.

- Los asesores de atención a la diversidad los Servicios Provinciales de la comunidad autónoma.

- Tres asesores de la Dirección General de Política Educativa y Educación Permanente, pertenecientes al Servicio de Innovación y Orientación educativa.

Fruto de este seminario, se organizaron en el mes de junio de 2013, las Primeras Jornadas 
Autonómicas de Educación Inclusiva, con los objetivos de acercar la educación inclusiva a todos los docentes, conocer metodologías que permitan dinamizar en los centros educativos políticas, culturas y prácticas inclusivas, así como promover una formación permanente del profesorado desde el enfoque de la educación inclusiva.

\section{d) Potenciar la formación on line por medio de aula Aragón}

Aragón es una comunidad con una superficie de $37.719 \mathrm{~km}^{2}$, que representa un 9,4\% del territorio de España. En el censo del año 2012 hay algo más de un millón trescientos mil habitantes, lo que representa una densidad de población de 28,27 y que se distribuye fundamentalmente en la ribera del río Ebro, existiendo zonas escasamente pobladas.

AulaAragón, un proyecto del Departamento de Educación, Universidad, Cultura y Deporte del Gobierno de Aragón, nace en el curso escolar 2002/2003 con la finalidad de desarrollar cursos de formación a distancia a través de Internet. La incorporación de las tecnologías de la información y la comunicación a la formación a distancia extienden las oportunidades de educación a sectores de la población hasta ahora excluidos por falta de disponibilidad horaria o debido a su lugar de residencia.

EI CAREI utiliza esta plataforma aragonesa de formación on line para llegar a todo el profesorado aragonés repartido en distintos lugares de la geografía aragonesa.

\section{e) Innovación}

Todos los años, el Departamento de Educación, Universidad, Cultura y Deporte del Gobierno de Aragón, convoca a todos los centros sostenidos con fondos públicos de niveles no universitarios para participar en el desarrollo de proyectos de Innovación e Investigación Educativa. Los proyectos que presenten los centros deben facilitar la puesta en práctica de experiencias que permitan concretar el marco teórico en que se fundamenta la innovación y la investigación educativa con la realidad de los centros y de las aulas de enseñanza, y deben referirse, preferentemente, a temas tales como mejora del proceso enseñanza-aprendizaje; mejora de la organización escolar; innovación en el desarrollo de programaciones de las áreas y materias del currículo, orientadas a la adquisición y evaluación de las competencias básicas, en las que se hagan explícitas las metodologías docentes a emplear; fomento de la competencia digital y del tratamiento de la información en las diferentes áreas y materias; procesos didácticos y organizativos enfocados a la atención de todo el alumnado como medida para garantizar el éxito escolar; la creación de comunidades de aprendizaje que puedan implicar a los distintos sectores de la comunidad educativa o cualquier experiencia innovadora que fomente el espíritu emprendedor.

Desde el CAREI se asesora y apoya a los centros docentes y al profesorado participante en el desarrollo de proyectos relacionados con la educación inclusiva, ofreciendo recursos materiales y humanos, así como formación especializada si fuese necesario. Pero corresponde a la inspección educativa supervisar la implantación, desarrollo y evaluación de los programas de Innovación e Investigación Educativa dentro del proceso de supervisión general de su organización y funcionamiento. 


\section{Trabajos citados}

AINSCOW, M. (2005). La respuesta a las necesidades educativas especiales en la escuela vasca inclusiva. Desarrollo de sistemas educativos inclusivos (págs. 19-36). Bilbao: Gobierno Vasco.

BLANCO, R. (2008). Conferencia Internacional de educación. La educación inclusiva: el camino hacia el futuro. Marco conceptual sobre educación inclusiva (págs. 5-14). Ginebra: UNESCO.

EUROPEA, C. (2012). Comunicación de la comisión al Parlamento Europeo, al Consejo, al Comité Económico y Social europeo y al Comité de las regiones. Estrasburgo: Comisión Europea.

EURYDICE. (2012). Developing Key competences at school in Europe. Challenges and opportunities for Policy. Brussels: Education, Audiovisual and Culture Executive Agency (EACEA).

MIR, M.; BATLE, M.; HERNÁNDEZ, M. (2009). Contexto de colaboración familia-escuela durante la primera infancia. Revista electrónica de investigación e innovación educativa y socioeducativa, $1 ., 44-68$.

MUNTANER, J. (2012). Tecnología e inclusión en el ámbito educativo, laboral y comunitarios. Recuperado el Marzo de 2013, de http://diversidad.murciaeduca.es/tecnoneet/2010/index.html

UNESCO. (2006). Orientaciones para la Inclusión: asegurar el acceso a la educación para todos. París: UNESCO.

UNESCO. (2009). Educación inclusiva: el camino hacia el futuro. Ginebra.

Submetido em 15 de agosto de 2013.

Aprovado em 04 de setembro de 2013.

Coral Elizondo Carmona: Directora del Centro Aragonés de Recursos para la Educación Intercultural del Departamento de Educación del Gobierno de Aragén - CAREI - Zaragoza - Espanha.

E-mail: coralelizondo@gmail.com 